\title{
Sepsis mit Purpura Fulminans nach Hundebiss*
}

\author{
Septic Shock and Purpura Fulminans after a Dog Bite
}

Autoren

Institute

\author{
K. Völl ' , G. Haase², H. Fritz ${ }^{1}$, J. Riebe ${ }^{3}$, C. Huszka ${ }^{4}$, J. Kindler ${ }^{1}$
}

Klinik für Innere Medizin, Medizinisches Zentrum Kreis Aachen gGmbH

Institut für Medizinische Mikrobiologie, Rheinisch-Westfälische Technische Hochschule Aachen

Praxis für Laboratoriumsmedizin, Aachen

Institut für Pathologie, Rheinisch-Westfälische Technische Hochschule Aachen

\section{Bibliografie}

DOI $10.1055 / \mathrm{s}-2007-995730$

Akt Dermatol 2008; 34:

236-239 @ Georg Thieme

Verlag KG Stuttgart · New York ISSN 0340-2541

Korrespondenzadresse Prof. Dr. med. Joachim Kindler Chefarzt Klinik für Innere Medizin, Medizinisches Zentrum Kreis Aachen $\mathrm{gGmbH}$ Mauerfeldchen 25 52146 Würselen joachim.kindler@mz-ac.de

\section{Zusammenfassung $\nabla$}

Anamnese und klinischer Befund: Ein 61-jähriger Patient stellte sich mit seit 4 Tagen zunehmenden Rückenschmerzen und abdominellen Beschwerden vor. Seit dem Vortag sei es zu einer Verschlechterung des Allgemeinzustandes mit Schwächegefühl und Dyspnoe gekommen. Bei Aufnahme war der Patient tachykard und tachypnoeisch mit Lippen- und Akrozyanose bei einer Körpertemperatur von $39,1^{\circ} \mathrm{C}$. Bis auf eine epigastrische Druckdolenz war der abdominelle Untersuchungsbefund unauffällig. Die gesamte Haut des Patienten verfärbte sich livide, und es entwickelten sich zunehmende Einblutungen mit zentralen Nekrosen. Fremdanamnestisch war eine Hundebissverletzung einige Wochen vor Aufnahme eruierbar.

Untersuchungen: Laborchemisch zeigte sich eine deutliche Entzündungskonstellation und Thrombozytopenie. Als Hinweis auf eine systemische bakterielle Infektion war die Procalcitonin-Konzentration im Serum signifikant erhöht. Röntgen-Thorax- sowie CT-Untersuchungen des Abdomens und der basalen Lungenabschnitte erbrachten keine richtungsweisenden Befunde. Im transösophagealen Echokardiogramm konnte eine Endokarditis ausgeschlossen werden, die Pumpfunktion war jedoch global stark reduziert. Die anaerobe Blutkultur ergab das Wachstum eines gramnegativen, pleomorphen Stäbchenbak-

\section{Kasuistik}

$\nabla$

\section{Anamnese}

Ein 61-jähriger Patient berichtete über seit einigen Tagen bestehende Rückenschmerzen und abdominelle Beschwerden. Seit dem Vortag sei es zu einer deutlichen Verschlechterung des Allgemeinzustandes mit einem körperlichen Schwächegefühl, zunehmender Luftnot und Fieber ge- teriums, das sich durch weitere molekulargenetische Untersuchungen als Capnocytophaga canimorsus identifizieren ließ.

Diagnose, Therapie und Verlauf: Der Patient wurde auf die Intensivstation aufgenommen. Bei einer Sepsis zunächst ohne eindeutigen Erreger oder Fokus begannen wir eine intravenöse Behandlung mit Piperacillin/Tazobactam. Im Verlauf wurde die Antibiose bei fraglicher meningealer Beteiligung um Ceftriaxon erweitert. Im Rahmen der Verbrauchskoagulopathie wurden Blutprodukte und Plasmabestandteile transfundiert. Trotz supportiver Maßnahmen starb der Patient nach 4 Tagen im Multiorganversagen.

Folgerung: Nach Hunde- und Katzenbissverletzungen sollte als Auslöser einer Sepsis, vor allem bei Patienten mit Splenektomie oder Alkoholabusus, eine Capnocytophaga canimorsus-Infektion in Betracht gezogen werden. Ein mikroskopischer Direktnachweis eines gramnegativen Stäbchenbakteriums im Blutausstrich kann diesen Verdacht erhärten, bevor dieser langsam wachsende Erreger kulturell gezüchtet worden ist. Bei der beschriebenen hohen Mortalität ist eine frühzeitige spezifische antibiotische Therapie von entscheidender Bedeutung für den weiteren Verlauf. Das Bewusstsein von Ärzten und Patienten sollte hinsichtlich der Infektionsgefährdung durch Bissverletzungen geschärft werden. Wie dieser Fall belegt, sollte nicht nur bei Risikopatienten eine Antibiotikaprophylaxe erfolgen.

kommen. Fremdanamnestisch war eine Hundebissverletzung an der linken Hand wenige Wochen vor dem Beginn der Beschwerden zu eruieren. Die oberflächliche Verletzung sei ohne medizinische Versorgung verheilt.

\footnotetext{
Erstveröffentlichung in: Dtsch Med Wochenschr 2007; 132: $1321-1324$.
} 


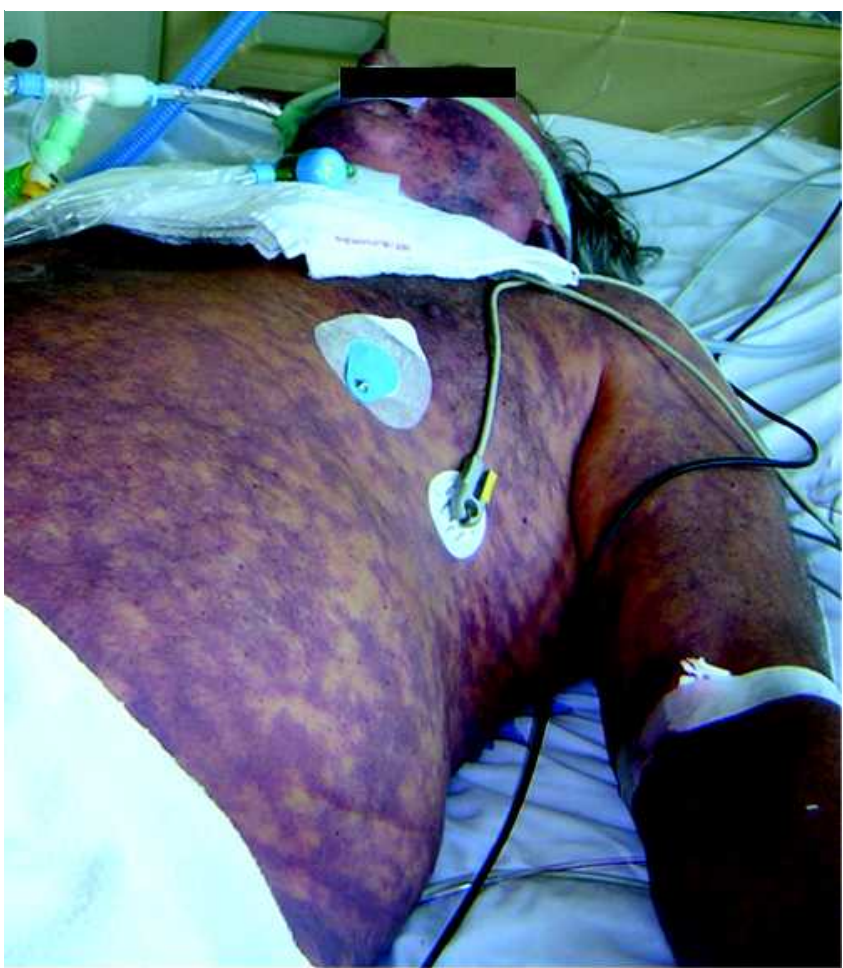

Abb. 1 Purpura fulminans bei disseminierter intravasaler Gerinnung.

Der Patient gab einen Nikotinabusus von ca. 30 pack years an. Allergien waren nicht bekannt. Zuletzt habe er regelmäßig Acetylsalicylsäure $100 \mathrm{mg} /$ die und Diclofenac eingenommen. An Vorerkrankungen wurden eine Hyperlipoproteinämie, Hyperurikämie, Adipositas $\left(180 \mathrm{~cm}, 100 \mathrm{~kg}\right.$, BMI $\left.31 \mathrm{~kg} / \mathrm{m}^{2}\right)$, Coxarthrose, Gonarthrose und Refluxösophagitis genannt. Anamnestisch ergab sich kein Anhalt für eine Immunsuppression.

\section{Körperlicher Untersuchungsbefund}

Der Patient war in akut reduziertem Allgemeinzustand mit Lippen- und Akrozyanose sowie Tachypnoe. Die Körpertemperatur lag bei $39,1^{\circ} \mathrm{C}$, der Blutdruck betrug $130 / 90 \mathrm{mmHg}$ bei einer Herzfrequenz von 140 Schlägen/min. Der abdominelle Untersuchungsbefund war bis auf einen diskreten epigastrischen Druckschmerz unauffällig. Es ließen sich einzelne Rasselgeräusche über den basalen Lungenabschnitten auskultieren. An den Extremitäten war eine livide Marmorierung zu erkennen, die sich im Verlauf auf den Stamm ausdehnte ( $\bullet$ Abb. 1). Initial zeigten sich keine fokal-neurologischen Defizite. Es ließ sich jedoch in den folgenden Stunden eine zunehmende situative Desorientiertheit und Vigilanzminderung feststellen.

\section{Klinisch-chemische Untersuchungen}

Die Entzündungs- und Sepsisparameter (C-reaktives Protein $234 \mathrm{mg} / \mathrm{l}$; Procalcitonin-Konzentration > $10 \mathrm{ng} / \mathrm{ml}$ - semiquantitativer Test - PCT-Q rapid, BRAHMS Aktiengesellschaft, Hennigsdorf, Germany) waren bei Aufnahme erhöht. Bei einer Gesamt-

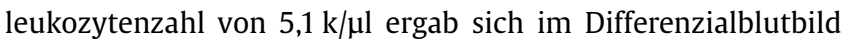
eine Neutrophilie (92\%). Als Zeichen einer beginnenden Verbrauchskoagulopathie (DIC=disseminierte intravasale Gerin-

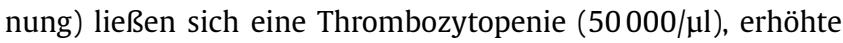
PTT (57 sec.) sowie erniedrigte Quick- (47\%) und ATIII-Konzentrationen (69\%) ermitteln. Des Weiteren fiel eine Erhöhung von

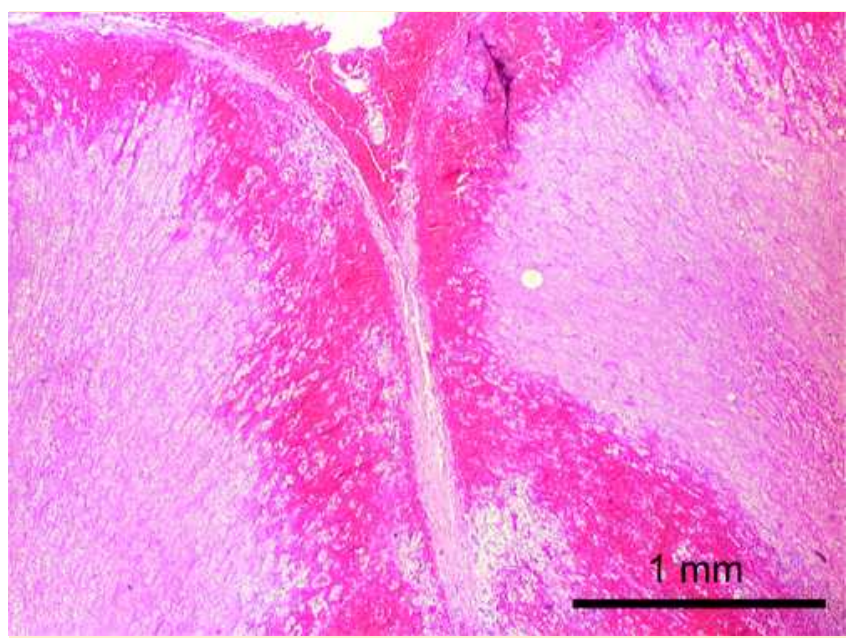

Abb. 2 Lichtmikroskopie der Nebenniere mit Einblutungen. Hämatoxylin-Eosin-Färbung.

Kreatinin (2,4 mg/dl), LDH (587 U/l), gGT (97 U/l) und Bilirubin gesamt $(3,1 \mathrm{mg} / \mathrm{dl})$ auf.

\section{Ergänzende Untersuchungen}

In einem von mehreren Blutkulturpaaren konnte nach 48 Stunden ein Bakterium kultiviert werden. Es ließ sich jedoch mit den üblichen phänotypischen Tests keinem der typischen Sepsiserreger zuordnen. Das Isolat wurde daher molekulargenetisch durch eine Analyse der 16S rDNA als Capnocytophaga canimorsus identifiziert [9].

Die Röntgen-Thorax-Untersuchung und die abdominelle CT mit Darstellung der basalen Lungenabschnitte ergaben keine richtungsweisenden Befunde. Entzündliche Prozesse konnten nicht dargestellt werden. Transthorakale und transösophageale Echokardiographie zeigten normgroße rechte und vergrößerte linke Herzhöhlen und eine global schwer reduzierte Pumpfunktion. Herzklappenvegetationen konnten ausgeschlossen werden.

\section{Therapie und Verlauf}

Nach der Abnahme von Blutkulturen wurde eine kalkulierte antibiotische Therapie mit Piperacillin/Tazobactam begonnen. Bei einer zunehmenden Vigilanzminderung und dem Verdacht einer meningealen Infektion wurde wegen der guten Liquorgängigkeit zusätzlich Ceftriaxon verabreicht. Im weiteren Verlauf kam es zu einer respiratorischen und kardiozirkulatorischen Insuffizienz, einem akuten Nierenversagen und einer disseminierten intravasalen Gerinnung. Es erfolgte eine differenzierte Katecholamin- und Volumentherapie sowie eine kontinuierliche Gabe von Hydrocortison (200 mg/die). Es wurden Erythrozytenund Thrombozytenkonzentrate transfundiert. Wenige Stunden nach der stationären Aufnahme wurde eine maschinelle Beatmung und eine kontinuierliche veno-venöse Hämofiltation mit hohen Substratmengen begonnen. Der Patient starb 2 Tage später an einem progredienten Multiorganversagen mit Ausbildung eines Acute Respiratory Distress Syndrome (ARDS).

Die Obduktion ergab makroskopisch und mikroskopisch Zeichen eines septischen Schocks und einer Verbrauchskoagulopathie mit Nachweis einer generalisierten Purpura, petechialen Hautläsionen, Nebenniereneinblutungen ( $\bullet$ Abb. 2), Schocknieren mit Ödembildung und hyalinen Zylindern sowie einer Milz von zerfließlicher Konsistenz. In der neuropathologischen Unter- 
suchung zeigte sich eine diskrete meningeale Reizung ohne Hinweis auf eine Meningitis. Ein Keimnachweis aus dem Liquor gelang nicht. Ein Ausgangsherd für die Sepsis ließ sich nicht identifizieren. Zeichen einer frischen Bissverletzung konnten nicht mehr nachgewiesen werden.

\section{Diskussion}

Die vorliegende Kasuistik beschreibt eine letale Capnocytophaga canimorsus-Infektion nach einer Hundebissverletzung. C. canimorsus wurde erstmals 1976 als Dysgonic-Fermenter (CDC group DF2)-Erreger einer Septikämie mit Meningitis beschrieben [2]. Von Brenner et al. wurde die Bezeichnung Capnocytophaga canimorsus eingeführt [3]. Capnocytophaga canimorsus ist ein gramnegatives, pleomorphes, anspruchsvolles Stäbchenbakterium mit langsamem Wachstum unter mikroaerophilen (capnophilen) Kulturbedingungen. Da Blutkulturen üblicherweise entweder nur unter aeroben bzw. anaeroben Bedingungen bebrütet werden, ist aufgrund der obligaten Capnophilie dieses Erregers der kulturelle Nachweis häufig nicht möglich oder das Wachstum unter anaeroben Bedingungen, wie in diesem Fall, stark verzögert. Die phänotypische Identifizierung wird zudem durch die geringe biochemische Aktivität (= dysgonic) in den üblicherweise benutzten Tests erschwert. Bei der Gramfärbung eines peripheren Blutausstrichs lassen sich jedoch für den Geübten frühzeitig Hinweise auf diesen Mikroorganismus gewinnen, da er bei einer Sepsis in sehr hohen Keimzahlen vorliegt und somit dort direkt sichtbar sein kann [5]. Es zeigen sich 2-4 $\mu$ m lange, feine, fusiforme Stäbchen, die gehäuft paarweise auftreten. Neben dem extrazellulärem Vorkommen ist in einigen Fällen nach Phagozytose ein intrazelluläres Vorliegen in neutrophilen Granulozyten beschrieben worden [1]. Eine sichere Identifizierung des Erregers ist durch Amplifikation des 16S rRNA-Gens mittels Polymerase-Ketten-Reaktion (PCR) und anschließender Identifizierung durch Sequenzvergleich möglich.

C. canimorsus ist Teil der normalen Mund- und Rachenflora von Hunden (24\%) und Katzen (17\%) [13]. Humane Infektionen kommen größtenteils nach Hundebiss- oder Kratzverletzungen vor. Krankheitszeichen treten in der Regel 2-3 Tage nach der Inokulation auf, es sind jedoch auch wie im vorliegenden Fall Inkubationszeiten von 2-4 Wochen beschrieben [7]. Der Krankheitsverlauf ist sehr variabel. Neben lokalen, problemlos verheilenden Infektionen sind in der Literatur bislang ca. 130 Fälle von Septikämien mit oftmals fulminantem letalen Verlauf bekannt. Dabei kommt es teilweise zu einer dem Toxic-Shock-Syndrom ähnlichen Symptomatik mit disseminierter intravasaler Gerinnung und akutem Nierenversagen. Es sind jedoch auch Assoziationen mit Meningitis, Endokarditis und infektiöser Arthritis aufgetreten [11]. Die Gesamt-Mortalität wird zwischen 23\% und $31 \%$ angegeben. Als Risikofaktoren für eine systemische Infektion gelten immunsuppressive Therapie, Asplenie, chronischer Alkoholismus, chronische Lungenkrankheiten, hämatologische Neoplasmen und Lebererkrankungen. Als klassische Therapie wird Penicillin G eingesetzt. Aufgrund der steigenden Prävalenz von Beta-Laktamase-produzierenden Stämmen wird mittlerweile jedoch eine breitere antibiotische Therapie mit Amoxicillin/Clavulansäure empfohlen. Alternativ können Drittgenerations-Cephalosporine, Imipenem, Clindamycin, Makrolide oder Tetrazyklin-Präparate eingesetzt werden. Resistenzen wurden für Aminoglykoside, Aztreonam und Cotrimoxazol beschrieben. Im vorliegenden Fall konnte keiner der beschriebenen
Risikofaktoren identifiziert werden. Als Ursache für die Infektion wird eine banale Hundbissverletzung angenommen. Trotz einer sofort eingeleiteten adäquaten antibiotischen Therapie verlief die Erkrankung letal, da es zu diesem Zeitpunkt bereits zu einer Initiierung der Sepsiskaskade mit irreversiblem Organversagen gekommen war.

In Anbetracht der Möglichkeit eines fulminanten septischen Krankheitsverlaufs mit hoher Letalität ist nach Hundebissverletzungen neben der lokalen Wundbehandlung und der ggf. durchzuführenden Tetanus- und Rabiesimmunisierung eine Antibiotikaprophylaxe zu erwägen. Der generelle Einsatz einer antibiotischen Therapie nach Hundebissverletzungen wird kontrovers diskutiert, ein eindeutiger Nutzen konnte bislang nicht belegt werden $[4,8]$. Bei hohem Risiko (z. B. Immunsuppression, Diabetes mellitus, Asplenie, Leberzirrhose) und bei besonderen Wundverhältnissen (Kopf- , Nacken- , Genital- , Hand- und Unterarmverletzungen, Quetsch- und Punktionswunden, starke Gewebedestruktion) wird jedoch eine 3-10-tägige Therapie mit Amoxicillin/Clavulansäure empfohlen $[6,10,12]$.

\section{Fazit \\ $\nabla$}

Bei der Sepsisbehandlung sind seltene Erreger und Infektionsquellen in Betracht zu ziehen. Um rasch eine gezielte antibiotische Therapie einleiten zu können, ist die Identifikation des Erregers entscheidend. Da der kulturelle Nachweis verschiedener Organismen scheitern kann, sollte frühzeitig die Gramfärbung und Mikroskopie eines Blutausstriches durchgeführt werden. Es ist Konsens, dass nach einer Hundebissverletzung bei Risikopatienten oder ungünstigen Wundverhältnissen eine Antibiotikaprophylaxe mit Amoxicillin/Clavulansäure durchgeführt werden sollte. Da jedoch mehrere Fälle beschrieben sind [7], in denen es ohne das Vorliegen von Risikofaktoren zu einem schweren Krankheitsverlauf gekommen ist, empfehlen wir eine prophylaktische antibiotische Therapie bei allen Hundebissverletzungen, die einen Hautdefekt zur Folge haben. Hierbei kommt es besonders auf den frühzeitigen Einsatz z.B. von Penicillinen mit $\beta$-Lactamaseinhibitor bereits bei der Wundversorgung an.

\section{Konsequenz für Klinik und Praxis}

Bei einer Sepsis nach einem Hunde- oder Katzenbiss sollte vor allem bei Risikopatienten auch an eine C. canimorsusInfektion gedacht und eine Gramfärbung eines Blutausstriches vorgenommen werden.

Entscheidend ist die Einleitung einer Antibiotikatherapie schon während der ersten Wundversorgung.

Autorenerklärung: Die Autoren erklären, dass sie keine finanziellen Verbindungen mit einer Firma haben, deren Produkt in dem Artikel eine wichtige Rolle spielt (oder mit einer Firma, die ein Konkurrenzprodukt vertreibt). 


\section{Abstract}

\section{Septic Shock and Purpura Fulminans after a Dog Bite $\nabla$}

History and clinical findings: A 61-year-old man presented with a four-day history of back pain and nonspecific abdominal pain. His condition had significantly worsened since the day before admission with generalized weakness and dyspnea. His temperature was $39.1^{\circ} \mathrm{C}$, he had tachycardia and was tachypneic. Peripheral cyanosis was noted. The abdomen was soft with mild epigastric tenderness. A diffuse skin rash developed with increasing petechial bleeding and central necrosis. It was revealed that he had been bitten by a dog several weeks before admission.

Investigations: Laboratory data indicated an acute inflammatory process with a marked increase in white blood cells and C-reactive protein. An elevated procalcitonin level suggested a systemic bacterial infection. Chest X-ray and abdominal CT scan were unremarkable. Echocardiography revealed a globally hypokinetic heart with no evidence of valvular vegetations. One set of blood cultures grew micro-aerophilic, Gram-negative rods. Gene sequencing identified the slow growing, fastidious bacillus as Capnocytophaga canimorsus.

Treatment and course: The patient was admitted to the intensive care unit and initially treated with intravenous piperacillin/ tazobactam and hydrocortisone for septic shock. Transfusions of platelets and blood products were given because of disseminated intravascular coagulation. The patient developed multi-organ failure requiring ventilation and hemodialysis; he died four days after admission.

Conclusions: As a rare cause of septicemia, especially in immunocompromised patients, Capnocytophaga canimorsus infection should be considered after an animal bite. Given the slow growth of this bacterium in culture, Gram-staining of a peripheral blood smear may provide an early diagnosis and avoid delay before appropriate antibiotic therapy, which may favorably influence the potentially fatal course, if started.

\section{Literatur}

1 Alberio L, Lammle B. Images in clinical medicine. Capnocytophaga canimorsus sepsis. N Engl J Med 1998; 339: 1827-1827

2 Bobo RA, Newton EJ. A previously undescribed gram-negative bacillus causing septicemia and meningitis. Am J Clin Pathol 1976; 65: 564 569

3 Brenner DJ, Hollis DG, Fanning GR et al. Capnocytophaga canimorsus sp. nov. (formerly CDC group DF-2), a cause of septicemia following dog bite, and C. cynodegmi sp. nov., a cause of localized wound infection following dog bite. J Clin Microbiol 1989; 27: 231 - 235

4 Cummings $P$. Antibiotics to prevent infection in patients with dog bite wounds: a meta-analysis of randomized trials. Ann Emerg Med 1994; 23: $535-540$

5 Janda JM, Graves MH, Lindquist D, Probert WS. Diagnosing Capnocytophaga canimorsus infections. Emerg Infect Dis 2006; 12: 340-342

6 Kuntz P, Pieringer-Müller E, Hof H. Infektionsgefährdung durch Bissverletzungen. Dtsch Arztebl 1996; 93: A969-972

7 Lion C, Escande F, Burdin JC. Capnocytophaga canimorsus infections in human: Review of the literature and cases report. Eur J Epidemiol 1996; $12: 521-533$

8 Medeiros I, Saconato H. Antibiotic prophylaxis for mammalian bites. Cochrane Database Syst Rev 2001; 2: CD001738 - CD001738

9 Meybeck A, Aoun N, Granados D et al. Meningitis due to Capnocytophaga canimorsus: Contribution of 16S RNA ribosomal sequencing for species identification. Scan J Infect Dis 2006; 38: 375 - 377

10 Morgan M, Palmer J. Dog bites. Brit Med J 2007; 334: 413-417

11 Pers C, Gahrn-Hansen B, Frederiksen W. Capnocytophaga canimorsus septicemia in Denmark, 1982 -1995: review of 39 cases. Clin Infect Dis 1996; 23: $71-75$

12 Stucker FJ, Shaw GY, Boyd S, Shockley WW. Management of animal and human bites in the head and neck. Arch Otolaryngol Head Neck Surg 1990; 116: 789-793

13 Westwell AJ, Kerr K, Spencer MB, Hutchinson DN. DF-2 infection. Brit Med J 1989; 298: 116- 117 